\title{
A population-based competing risk survival analysis of patients with salivary duct carcinoma
}

\author{
Jianchuan $\operatorname{Ran}^{1,2}$, Huihui Zou ${ }^{1,2}$, Xiaoye Li $^{1,2}$, Feng Guo ${ }^{1,2}$, Wenguang Xu ${ }^{1,2}$, Wei Han ${ }^{1,2}$ \\ ${ }^{1}$ Department of Oral and Maxillofacial Surgery, Nanjing Stomatological Hospital, Medical School of Nanjing University, Nanjing, China; ${ }^{2}$ Central \\ Laboratory of Stomatology, Nanjing Stomatological Hospital, Medical School of Nanjing University, Nanjing, China \\ Contributions: (I) Conception and design: W Han, W Xu; (II) Administrative support: None; (III) Provision of study materials or patients: J Ran, H \\ Zou; (IV) Collection and assembly of data: J Ran, H Zou; (V) Data analysis and interpretation: X Li, F Guo; (VI) Manuscript writing: All authors; (VII) \\ Final approval of manuscript: All authors. \\ Correspondence to: Wei Han; Wenguang Xu. Department of Oral and Maxillofacial Surgery, Nanjing Stomatological Hospital, Medical School of \\ Nanjing University, 30 Zhongyang Road, Nanjing, China. Email: doctorhanwei@hotmail.com; wenguang.xu@foxmail.com.
}

Background: Salivary duct carcinoma (SDC) is a rare malignancy with high risk of local recurrence and distant metastases of the salivary gland. This study was designed to summarize the clinical and pathological features and to further evaluate them as potential prognostic factors for SDC in the salivary gland.

Methods: Clinical data of 266 patients diagnosed with SDC between 2004 and 2015 were collected from the Surveillance, Epidemiology, and End Results (SEER) database. The prognostic factors affecting overall survival (OS) and cancer-specific survival (CSS) were determined by Kaplan-Meier analyses and Cox proportional hazards model. The nomogram was established to predict OS and CSS for SDC. The predictive accuracy of the nomograms was measured by concordance index (C-index).

Results: The 3- and 5-year OS of SDC patients were $67.41 \%$ and $47.86 \%$, while the 3- and 5-year CSS were $84.6 \%$ and $60.7 \%$, respectively. The primary site, $\mathrm{T}$ stage and $\mathrm{M}$ stage were identified as independent prognostic factors for OS by the multivariate analysis, whereas $\mathrm{N}$ stage, $\mathrm{M}$ stage, the presence of multiple primary carcinomas and the treatment modalities were identified as independent prognostic factors for CSS. The C-index values of the prognostic nomogram based the risk factors affecting SDC OS and CSS were 0.703 (0.646-0.760) and $0.771(0.691-0.851)$, respectively.

Conclusions: SDC is an aggressive malignancy with a high proportion of advanced stage and lymph node metastases. Patients with increasing age, submandibular gland malignancy, advanced T stage, advanced $\mathrm{N}$ stage, advanced $M$ stage, high lymph node ratio (LNR) and the presence of multiple primary carcinomas tend to have unfavorable outcomes. Radiotherapy or chemotherapy improve CSS remarkably. These factors will aid in effective therapeutic treatment modalities for SDC.

Keywords: Salivary duct carcinoma (SDC); Surveillance, Epidemiology, and End Results (SEER); competing risk analyses; nomogram, prognosis

Submitted Feb 23, 2020. Accepted for publication Sep 18, 2020.

doi: $10.21037 /$ atm-20-1849

View this article at: http://dx.doi.org/10.21037/atm-20-1849

\section{Introduction}

Salivary duct carcinoma (SDC) is a rare salivary gland malignancy, accounting for $35 \%$ of the salivary gland malignancies (1). SDC commonly occurs in the parotid gland and submandibular or minor salivary glands, among which the parotid gland is the most frequently involved site
$(2,3)$. Histologically resembling high-grade breast ductal carcinoma, SDC exhibits aggressive clinical features with a high rate of local recurrence and distant metastases, and thereby leading to poor clinical outcome (4). Previously, the clinical and pathological characteristics of SDC and their potentials as prognostic factors have been investigated (5-8). 
The most commonly identified prognostic factors of SDC include age, gender, tumor grade, tumor size, lymph node metastasis and clinical stage (9-12). Some other factors such as increasing numbers of positive lymph nodes, lymph node ratio (LNR), lymph-vascular invasion, perineural invasion and HER2/neu receptor expression were also shown to influence the prognosis of $\operatorname{SDC}(8,13,14)$. However, due to the rarity of this disease, most of those studies focused on single-center studies with limited sample size $(1,12,15,16)$. Therefore, the prognostic factors for SDC still remain to be largely elucidated.

To date, two large cohort studies with 495 SDC patients from 2004 to 2013 based on the National Cancer Database (NCDB) and with 228 SDC patients from 1973 to 2008 based on the Surveillance, Epidemiology, and End Results (SEER) database have been reported $(9,17)$. Given that NCDB does not provide data regarding cause of death, the first study based on NCDB that ignored the impact of survival from competing events might have led to misleading conclusions (9). Similarly, in the second study based on SEER that used SDC patient data without evaluating traditional the tumor, lymph node, metastasis (TNM) staging systems from a long period of time (1973 to 2008), the conclusions may also be incomprehensive since during this time the clinical and histopathological diagnostic criteria for SDC have evolved significantly (17).

In this population-based study, we analyzed the clinicopathologic characteristics and determined the prognostic factors for SDC of the salivary gland using SEER database. We also established a novel nomogram for predicting the overall survival (OS) and cancer-specific survival (CSS) of SDC patients, which could more precisely estimate the survival probability of individual SDC patients. We present the following article in accordance with the TRIPOD reporting checklist (available at http://dx.doi. org/10.21037/atm-20-1849) (18).

\section{Methods}

\section{Patients}

The study cohort of SDC patients from 2004 to 2015 were obtained from the SEER database (https://seer.cancer.gov/ data/) using SEER*Stat 8.3.6 software. Inclusion criteria were as follows. Firstly, pathological diagnosis of patients was SDC [International Classification of Diseases for Oncology, Third Edition (ICD-O-3) site code, histology code: 8500]. Secondly, the primary site was limited to the parotid gland and submandibular gland. Thirdly, the diagnosis year was from 2004 to 2015. Exclusion criteria included patients with unknown race, clinical stage based on the American Joint Committee on Cancer (AJCC) cancer staging or TNM system that was not clear and survival time that was less than 1 month. The study was conducted in accordance with the Declaration of Helsinki (as revised in 2013). Since any information in the SEER database does not require explicit consent from the patients, our study was not subject to the ethical approval requirements of the institutional review board.

\section{Study variables}

From SEER data, we extracted variables including race, sex, age at diagnosis, year of diagnosis, primary tumor site, laterality, histological grade, SEER summary stage, AJCC stage, T stage, $\mathrm{N}$ stage, $\mathrm{M}$ stage, surgery procedures, lymph node dissection, regional nodes examined positively, the presence of multiple primary carcinomas, adjuvant radiotherapy, chemotherapy, and follow-up information. The TNM stage were restaged according to the 7 th edition of AJCC staging system. LNR was calculated as the number of positive regional lymph nodes divided by the total number of lymph nodes examined. The LNR above or below the median level of LNR of all the SDC patients was defined as high or low, respectively. The survival time was measured as the time from the date of surgery to the last follow-up or to the death due to all causes (OS) or SDC (CSS).

\section{Statistical analyses}

Before modeling, categorical variables were grouped and the continuous variables were converted to the categorical variables to conform to the linear hypothesis. The OS and CSS estimation and survival curves were evaluated by Kaplan-Meier method and verified by log-rank test. Independent risk factors were determined by backward stepwise in Cox proportional risk (PH) regression model (19). Based on Cox $\mathrm{PH}$ regression results, a nomogram of all independent prognostic factors for combining 3- and 5-year OS predictions was constructed using the rms package in $\mathrm{R}$ software. The discrimination of nomogram was evaluated by Harrell's concordance index (C-index), which could estimate the probability between the observed and predicted OS and CSS (20). The calibration was evaluated using the calibration curve by comparing the estimated 
Table 1 Demographics of SDC of salivary gland

\begin{tabular}{|c|c|c|}
\hline \multirow{2}{*}{ Characteristics } & \multicolumn{2}{|c|}{ Patients $(n=266)$} \\
\hline & Number & $\%$ \\
\hline Age at diagnosis & 29-93 years o & .5 , median 68 ) \\
\hline \multicolumn{3}{|l|}{ Age group, years } \\
\hline$<50$ & 22 & 9.74 \\
\hline $50-59$ & 43 & 16.17 \\
\hline $60-69$ & 76 & 28.57 \\
\hline $70-79$ & 75 & 28.20 \\
\hline$>80$ & 50 & 22.12 \\
\hline \multicolumn{3}{|l|}{ Gender } \\
\hline Male & 189 & 71.05 \\
\hline Female & 77 & 28.95 \\
\hline \multicolumn{3}{|l|}{ Race } \\
\hline White & 215 & 80.82 \\
\hline Black & 27 & 10.15 \\
\hline Other & 24 & 9.03 \\
\hline \multicolumn{3}{|l|}{ Year of diagnosis } \\
\hline 2004-2010 & 121 & 45.49 \\
\hline 2011-2015 & 145 & 54.51 \\
\hline
\end{tabular}

SDC, salivary duct carcinoma.

risk line on the plot to the risk line observed on the axis. $\mathrm{R}$ version 3.6.1 software (The R Foundation for Statistical Computing, Shanghai, China. http://www.r-project.org), along with SPSS version 22 (SPSS Inc., Chicago, IL, USA), were employed to perform statistical analyses and plot the figures. A two-tailed $\mathrm{P}$ value $<0.05$ was considered statistically significant.

\section{Results}

\section{Demographics and clinicopathological characteristics of $S D C$}

A total of 266 patients were diagnosed with salivary gland SDC from 2004 to 2015. The median age was 68 (range, 29-93) years old, and the majority was male (71.05\%), with the highest incidence in the sixth decade of life (Figure S1). Of these patients, 215 (80.82\%) patients were white, 27 $(10.15 \%)$ patients were black and $24(9.03 \%)$ patients were from other ethnics (including American Indian/AK
Native, Asian/Pacific Islander). Among diagnosed cases, 121 (45.49\%) patients were diagnosed from 2004 to 2010, and 145 (54.51\%) patients were diagnosed from 2011 and 2015 (Table 1).

The most predominant primary site was the parotid gland (231 patients, $86.84 \%$ ), followed by the submandibular gland (35 patients, 13.16\%). According to the SEER summary stage, 63 (23.68\%) patients were at localized stage, $92(34.59 \%)$ patients were at regional stage, and $111(41.73 \%)$ patients were at distant metastasis stage. Based on the 7 th edition of AJCC TNM stage, the disease progressions were as follows: $54(20.30 \%) \mathrm{T} 1$ patients, $56(21.05 \%) \mathrm{T} 2$ patients, 77 (28.95\%) T3 patients, $72(27.07 \%) \mathrm{T} 4$ patients, and $7(2.63 \%)$ Tx patients. For lymph node metastasis, 100 (37.59\%) patients were at N0 stage, $160(60.15 \%)$ patients were at $\mathrm{N} 0+$ stages, and 6 $(2.26 \%)$ were $\mathrm{Nx}$ patients. In terms of distant metastasis, most of the patients $(90.97 \%)$ were at the M0 stage, while $24(9.02 \%)$ patients were at the M1 or Mx stage. As a whole, $64(24.06 \%)$ patients were at the early clinical stage (AJCC stage I and II) and $161(73.68 \%)$ patients were at the advanced clinical stage (AJCC stage III and IVA-C), suggesting that SDC is an aggressive malignancy in the salivary gland. The distribution of histological grade in the cohort was also centralized in a higher grade, among which $38.72 \%$ and $27.82 \%$ had grade III and grade IV disease, respectively. Finally, among multiple primary carcinomas collected, 175 (65.79\%) patients had the SDC and 91 (34.21\%) patients harbored other types of carcinomas, which led to competing risks for patient survival (Table 2).

\section{Treatment and outcome}

The majority of SDC patients (255 patients, $95.86 \%$ ) were treated surgically, while 9 patients were not recommended for surgery and 2 patients were recommended but refused. Among the 255 patients who received the surgery, neck dissection was performed in the majority of the patients $(228,89.80 \%)$. In 220 patients who underwent the neck dissection, examined lymph node data were available, which show that 151 patients had positive lymph nodes with the median number of 5 (range, 1-75). The number of positive regional lymph nodes against the total number of regional lymph nodes examined in the 220 patients are plotted in Figure 1A. The median LNR value was 0.167 . In terms of the overall treatment modalities, 60 patients were treated surgically only, 130 patients received the adjuvant radiotherapy after surgery, and 59 patients received adjuvant 
Table 2 Clinicopathological characteristics of SDC of salivary gland

\begin{tabular}{|c|c|c|}
\hline \multirow{2}{*}{ Characteristics } & \multicolumn{2}{|c|}{ Patients $(n=266)$} \\
\hline & Number & $\%$ \\
\hline \multicolumn{3}{|l|}{ Primary site } \\
\hline Parotid gland & 231 & 86.84 \\
\hline Submandibular gland & 35 & 13.16 \\
\hline \multicolumn{3}{|l|}{ Laterality } \\
\hline Left & 117 & 43.98 \\
\hline Right & 149 & 56.02 \\
\hline \multicolumn{3}{|l|}{ Summary stage } \\
\hline Localized & 63 & 23.68 \\
\hline Regional & 92 & 34.59 \\
\hline Distant & 111 & 41.73 \\
\hline \multicolumn{3}{|l|}{ AJCC stage } \\
\hline I & 37 & 13.91 \\
\hline II & 27 & 10.15 \\
\hline III & 35 & 13.16 \\
\hline IVA & 125 & 46.99 \\
\hline IVB & 10 & 3.76 \\
\hline IVC & 26 & 9.77 \\
\hline Unknow & 6 & 2.26 \\
\hline \multicolumn{3}{|l|}{ T stage } \\
\hline $\mathrm{T} 1$ & 54 & 20.30 \\
\hline $\mathrm{T} 2$ & 56 & 21.05 \\
\hline T3 & 77 & 28.95 \\
\hline $\mathrm{T} 4$ & 72 & 27.07 \\
\hline TX & 7 & 2.63 \\
\hline \multicolumn{3}{|l|}{$\mathrm{N}$ stage } \\
\hline NO & 100 & 37.59 \\
\hline N1 & 32 & 12.03 \\
\hline N2 & 123 & 46.24 \\
\hline N3 & 5 & 1.88 \\
\hline NX & 6 & 2.26 \\
\hline \multicolumn{3}{|l|}{ M stage } \\
\hline MO & 242 & 90.97 \\
\hline M1 & 20 & 7.52 \\
\hline$M X$ & 4 & 1.50 \\
\hline
\end{tabular}

Table 2 (continued)
Table 2 (continued)

\begin{tabular}{lcc}
\hline \multirow{2}{*}{ Characteristics } & \multicolumn{2}{c}{ Patients (n=266) } \\
\cline { 2 - 3 } Grade & \multicolumn{1}{c}{ Number } \\
Well differentiated & 6 & 2.26 \\
Moderately & 23 & 8.65 \\
differentiated & & \\
Poorly differentiated & 103 & 38.72 \\
Undifferentiated & 74 & 27.82 \\
Unknow & 60 & 22.56 \\
Multiple primary carcinomas & & 34.21 \\
Presence & 91 & 65.79 \\
Absence & 175 & \\
\hline $\begin{array}{l}\text { SDC, salivary duct carcinoma; } \\
\text { on Cancer. }\end{array}$ &
\end{tabular}

radiotherapy and chemotherapy. A few patients received no treatment or just received the adjuvant chemoradiotherapy or chemotherapy (Table 3). More specifically, among 64 early-stage (stage I \& II) SDC patients, only 19 (29.69\%) patients were treated surgically only, and 39 (60.94\%) patients received radiotherapy after surgery. Among a total of 196 late-stage (stage III \& IV) SDC patients, 38 (19.39\%) patients underwent surgery alone; $88(44.90 \%)$ patients were offered radiotherapy after surgery, 55 (28.06\%) patients received adjuvant radiotherapy and chemotherapy after surgery (Figure S2).

The median follow-up duration for all patients was 34.5 (range, 2-143) months. A total of 119 (44.74\%) patients died, among which 59 patients died of salivary gland malignancies and 60 patients died of other causes, including other malignant tumors and other types of systemic disease. The 3 - and 5 -year OS were $67.41 \%$ and $47.86 \%$, respectively, while the 3 - and 5-year CSS were $84.6 \%$ and $60.7 \%$, respectively (Figure 1B).

\section{Prognostic factors}

For OS analysis, patients with missing or uncertain values of clinical variables were excluded, and finally a total of 198 patients were included.

The univariate analysis revealed that SEER summary stage, T stage, $\mathrm{N}$ stage, $\mathrm{M}$ stage, AJCC clinical stage, surgery and LNR were negatively associated with OS. 

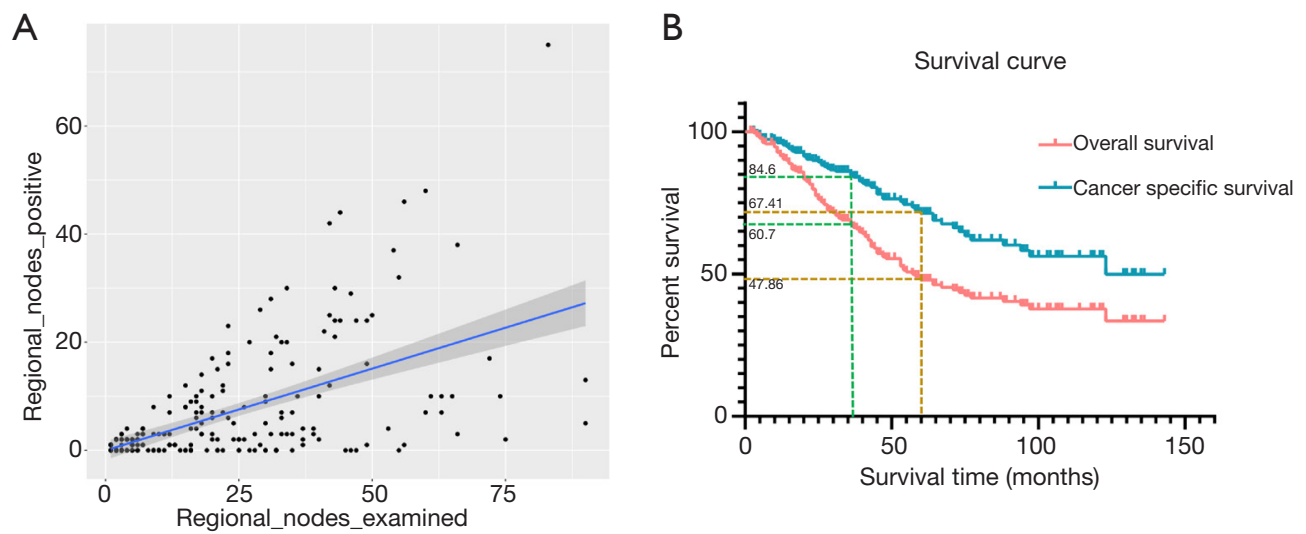

Figure 1 Lymph nodes positive and survival of SDC patients. (A) Positive regional lymph nodes are plotted against the total number of regional lymph nodes in SDC who underwent neck dissection; (B) Kaplan-Meier estimate for OS and CSS. SDC, salivary duct carcinoma; OS, overall survival; CSS, cancer-specific survival.

On the contrary, the OS rate was not influenced by the following clinical characteristics: age, gender, race, year of diagnosis, primary site, laterality, histological grade, the presence of multiple primary carcinomas, neck dissection and treatment modalities. The Kaplan-Meier analysis and log-rank test further visualized and validated the results (Figures 2 and Figure S3). The multivariate analysis identified the primary site, $T$ stage and $M$ stage as the negative prognostic factors. The results of the univariate and multivariate analysis for OS are shown in Table 4.

For CSS analysis, univariate analysis proved that the following factors had the significant influence on patients' survival: age at diagnosis, SEER summary stage, T stage, $\mathrm{N}$ stage, $\mathrm{M}$ stage and the presence of multiple primary carcinomas. The Kaplan-Meier curves further verified the results, and AJCC stage was also determined as a prognostic factor negatively affecting CSS (Figure 3 and Figure S4). Multivariate analysis showed that the independent prognostic factors for CSS were $\mathrm{N}$ stage, $\mathrm{M}$ stage, the presence of multiple primary carcinomas and treatment modalities. The results of the univariate and multivariate analyses for CSS are summarized in Table 5 .

The survival difference among patients treated with surgery alone and patients treated with surgery and adjuvant treatment was also evaluated. On univariate and multivariate analysis for OS, no significant difference was noted between patients treated surgically alone and patients who received surgery, adjuvant radiation and chemotherapy. Similar results were also observed when these treatment modalities were compared after stratified by AJCC stage of the malignancy (Figure S5). On univariate analysis for CSS, no significant difference was noted among patients treated with different modalities. However, on multivariate analysis for CSS, it was surprising that the prognosis of patients who received adjuvant radiation [hazard ratio (HR), 0.256; $\mathrm{P}=0.004]$ or adjuvant chemo-radiotherapy (HR, 0.217; $\mathrm{P}=0.007$ ) was better than the prognosis of patients treated with surgery only. This indicates that adjuvant radiation and chemotherapy improved the survival of SDC patients.

\section{Nomogram}

To visualize the multivariate results and improve the clinical practicality, the risk factors affecting OS and CSS included in the multivariate COX model were employed to construct a prognostic nomogram. Based on the total score identified, the nomograms can provide the likelihood of 3- and 5-year OS and CSS for individual SDC patient (Figure 4). The C-index value of the nomogram for OS and CSS was 0.703 (0.646-0.760) and $0.771(0.691-0.851)$, respectively. The calibration curve showed that the predicative curve fitted well with the actual observation curve, which indicated that there was a good agreement between the predicted 3- and 5 -year OS and CSS in the nomogram and the actual 3- and 5 -year OS and CSS in clinical practice (Figure 5).

\section{Discussion}

To our knowledge, the data collected in the present study represent the latest update from the SEER database on SDC of the salivary gland. Our results show that patient age, tumor size and lymph node metastasis negatively affect 
Table 3 Treatment and outcome of SDC of salivary gland

\begin{tabular}{|c|c|c|}
\hline \multirow{2}{*}{ Characteristics } & \multicolumn{2}{|c|}{ Patients (n=266) } \\
\hline & Number & $\%$ \\
\hline \multicolumn{3}{|l|}{ Surgery } \\
\hline Surgery & 255 & 95.86 \\
\hline Not recommended & 9 & 3.38 \\
\hline Recommended but refused & 2 & 0.75 \\
\hline \multicolumn{3}{|l|}{ Neck dissection } \\
\hline Yes & 228 & 85.71 \\
\hline None & 38 & 14.29 \\
\hline \multicolumn{3}{|l|}{ Regional nodes examined } \\
\hline Yes & 220 & 82.71 \\
\hline No & 37 & 13.91 \\
\hline Unknow & 9 & 3.38 \\
\hline \multicolumn{3}{|l|}{ Regional nodes positive $(\mathrm{n}=220)$} \\
\hline Positive & 151 & 68.64 \\
\hline Negative & 69 & 31.36 \\
\hline LNR & \multicolumn{2}{|c|}{ Median 0.167 , range $[0-1]$} \\
\hline \multicolumn{3}{|l|}{ Treatment modality } \\
\hline No treatment & 5 & 1.88 \\
\hline Surgery only & 60 & 22.56 \\
\hline Chemotherapy only & 2 & 0.75 \\
\hline Surgery + radiotherapy & 130 & 48.87 \\
\hline Surgery + chemotherapy & 6 & 2.26 \\
\hline Radiotherapy + chemotherapy & 4 & 1.50 \\
\hline $\begin{array}{l}\text { Surgery + radiotherapy + } \\
\text { chemotherapy }\end{array}$ & 59 & 22.18 \\
\hline Follow-up duration & \multicolumn{2}{|c|}{$\begin{array}{c}\text { Range } 2-143 \text { months, median } \\
34.5 \text { months }\end{array}$} \\
\hline \multicolumn{3}{|l|}{ Survival status } \\
\hline Alive & 147 & 55.26 \\
\hline Died of salivary gland & 59 & 22.18 \\
\hline Died of other causes & 60 & 22.56 \\
\hline
\end{tabular}

SDC, salivary duct carcinoma; LNR, lymph node ratio.

the prognosis of SDC. In addition, we also identified that the SEER summary stage, AJCC stage, LNR, primary site and the presence of multiple primary carcinomas are the important factors indicating poor prognosis for SDC, whereas histologic grade is not a prognostic factor, which might need further investigation in a large validation cohort. Overall, our study provided more clinical and survival aspects of SDC in comparison with the previous SEER study on SDC, including the 7th edition of TNM staging systems, LNR and presence of multiple primary carcinomas.

Previously, a study using NCDB to analyze the treatment patterns and the outcome data for patients with SDC in a large population-based sample $(n=495)$, showed that advanced stage, increasing age and male gender are associated with inferior OS (9). However, the NCDB used in the study did not provide data regarding the cause of death. As the non-cancer events contribute to a proportion of patient deaths, the competing risk events need to be taken into account in the survival analysis to reach a more objective conclusion. In our study, 59 (22.18\%) patients died of SDC while 60 (22.56\%) patients died of other causes. Accordingly, the 5-year OS and CSS were calculated as $47.86 \%$ and $60.7 \%$, respectively, which was consistent with that from the previous studies $(3,4,21)$.

In agreement with the existing literature indicating that most SDC patients were the men aged 50 or older $(2,17,22)$, and that the majority of tumors occurred in the parotid gland with advanced $\mathrm{T}$ stage $(3,6,23)$ (i.e., $\mathrm{T} 3$ or $\mathrm{T} 4)$ and regional lymph node metastasis $(\mathrm{N} 0+)$ (24), our analyses showed that SDC was most frequently seen in male patients within the sixth decade of life, and that $56.20 \%$ of tumors were at the advanced $\mathrm{T}$ stage and $60.15 \%$ of tumors were clinical $\mathrm{N} 0+$ disease. Accordingly, only $24.06 \%$ of patients presented at early clinical stage (AJCC stage I and II), whereas most of patients $(73.68 \%)$ presented at late clinical stage (AJCC stage III and IVA-C). Taken together, it suggests that SDC is an aggressive malignancy with advanced stage and high rate of lymph node metastasis.

In our study, the majority of SDC patients (95.86\%) underwent surgical resection and a high proportion $(89.80 \%)$ of them underwent neck dissection. This is in accordance with the National Comprehensive Cancer Network (NCCN) guidelines for high grade salivary malignancies (25). In comparison with previous studies concerning the survival benefit of adjuvant therapy for SDC, our study verified the role of radiotherapy and chemotherapy in improving the prognosis in patients with SDC, which might provide new support for the establishment of therapeutic guidelines for this disease $(9,15,17)$. However, more clinical trials are needed to further investigate the survival benefits of the addition of 
A
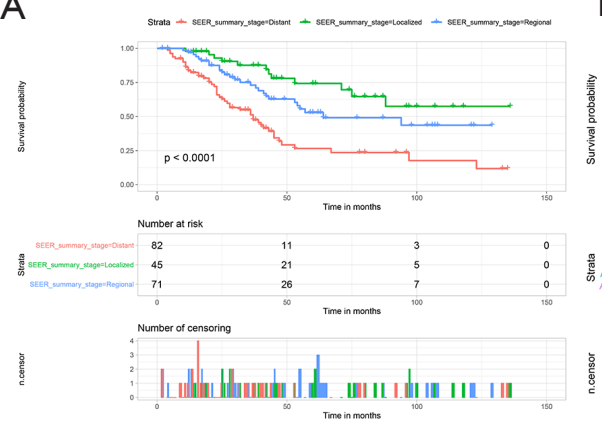

D
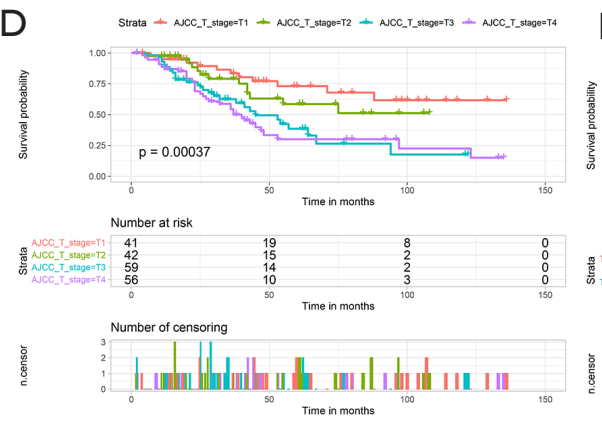

G

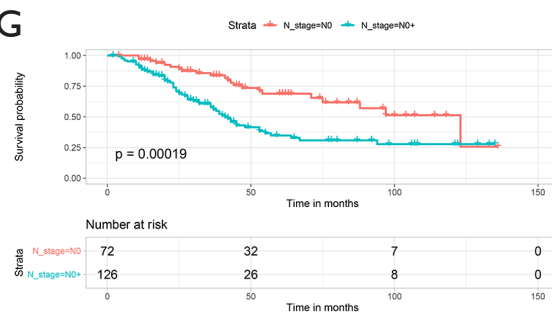

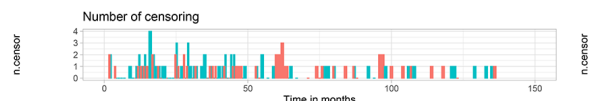
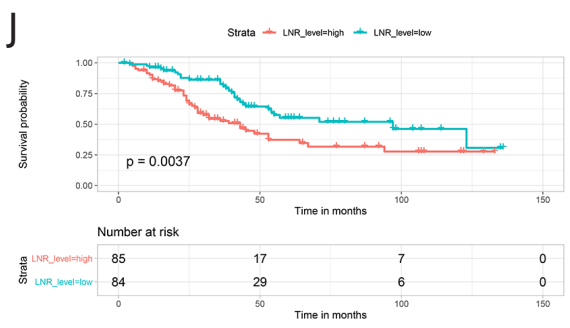

Number of censoring
B

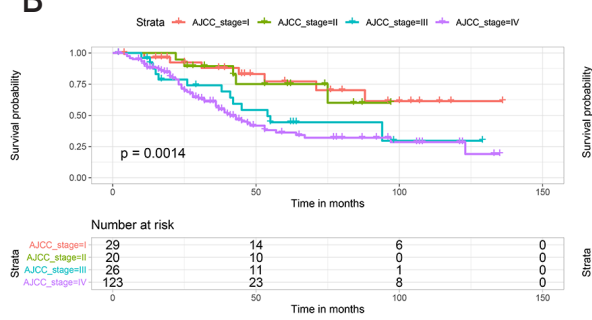

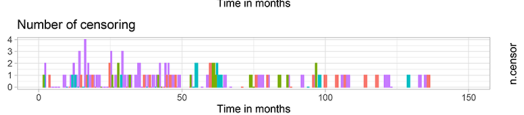

\section{E}
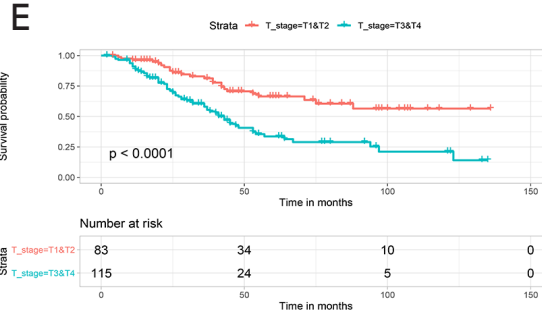

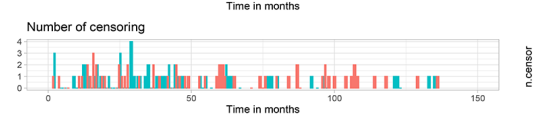

$\mathrm{H}$

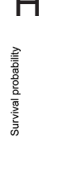

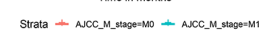

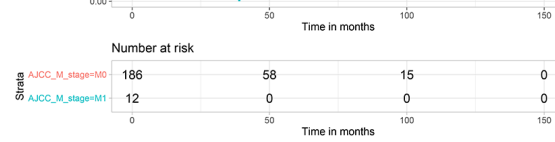

Number corsonging

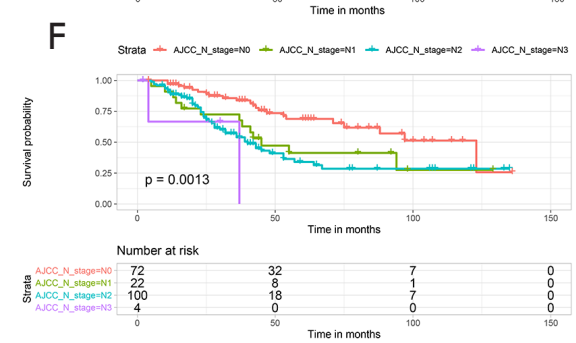

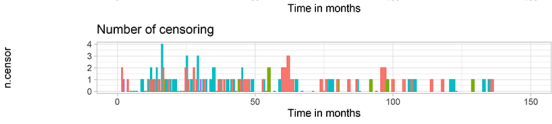

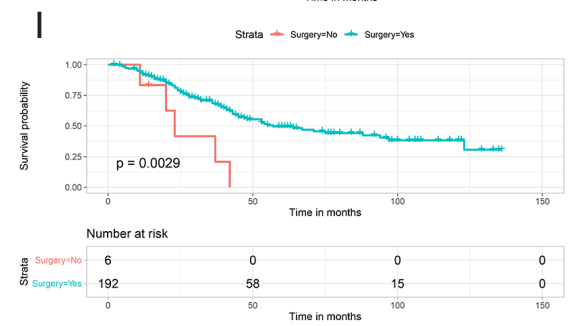

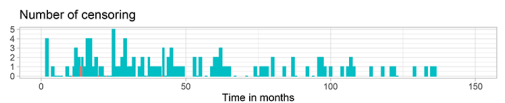

Figure 2 Kaplan-Meier curves for OS of SDC patients stratified by (A) SEER summary stage, (B,C) AJCC stage, (D,E) AJCC T stage, (F,G) AJCC N stage, (H) M stage, (I) surgery and (J) LNR level. OS, overall survival; SDC, salivary duct carcinoma; SEER, Surveillance, Epidemiology, and End Results; AJCC, American Joint Committee on Cancer; LNR, lymph node ratio.

radiation and chemotherapy.

To date, the commonly identified prognostic factors of SDC are as follows: age, gender, tumor grade, tumor size, lymph node metastasis and clinical stage (9-12). The prognostic factors determined in our study were similar to those expected based on prior studies. Furthermore, our multivariate analysis also showed that SDC originated at submandibular gland exhibited worse OS than that in the parotid gland, and that high LNR was a significant predictor of worse OS. Consistently, a retrospective study of the role 
Table 4 Univariate and multivariate survival analysis on OS for patients with SDC

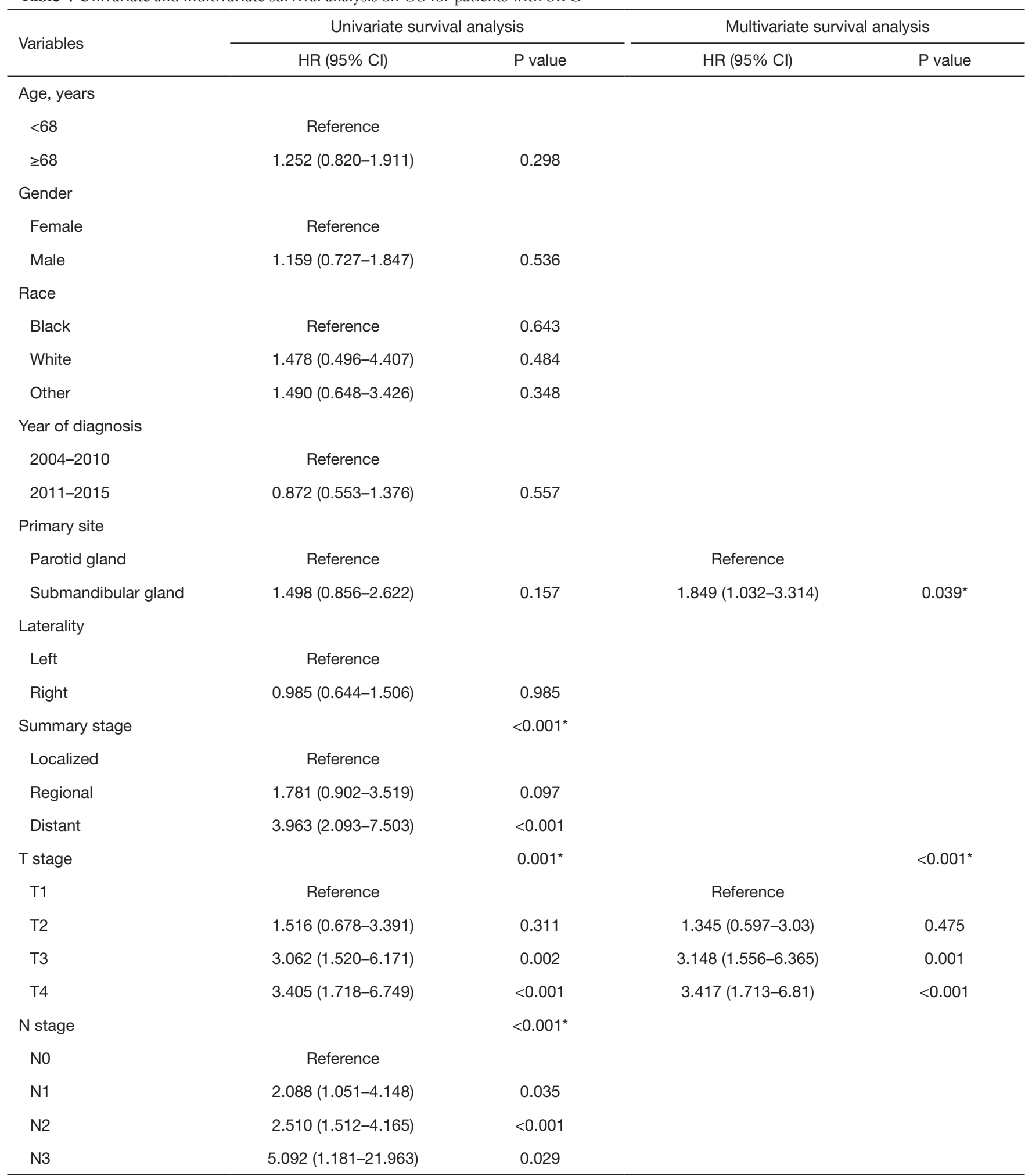

Table 4 (continued) 
Table 4 (continued)

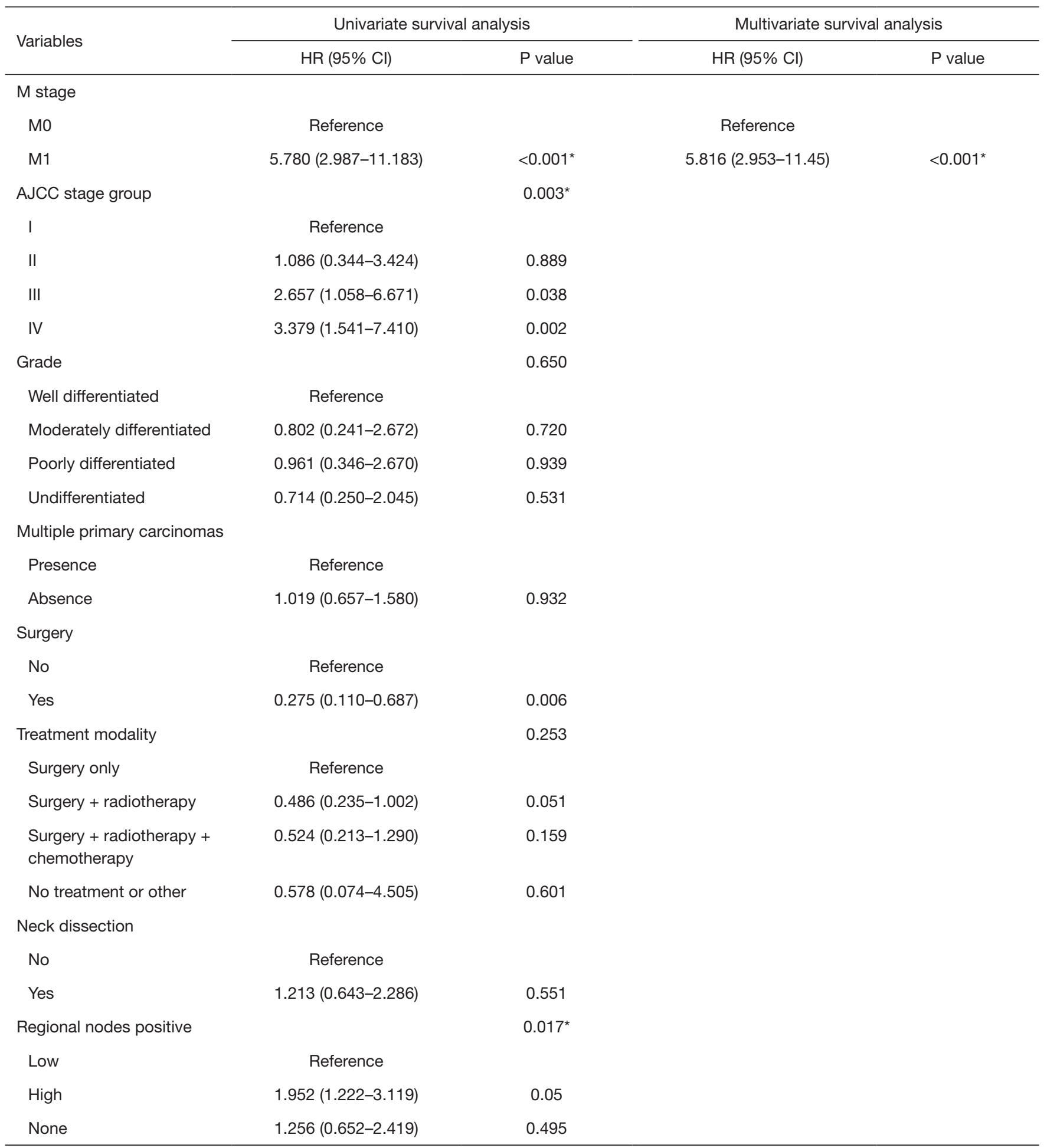

The number with * indicates statistical significance. OS, overall survival; SDC, salivary duct carcinoma; $\mathrm{HR}$, hazard ratio; $\mathrm{Cl}$, confidence interval; AJCC, American Joint Committee on Cancer. 
A
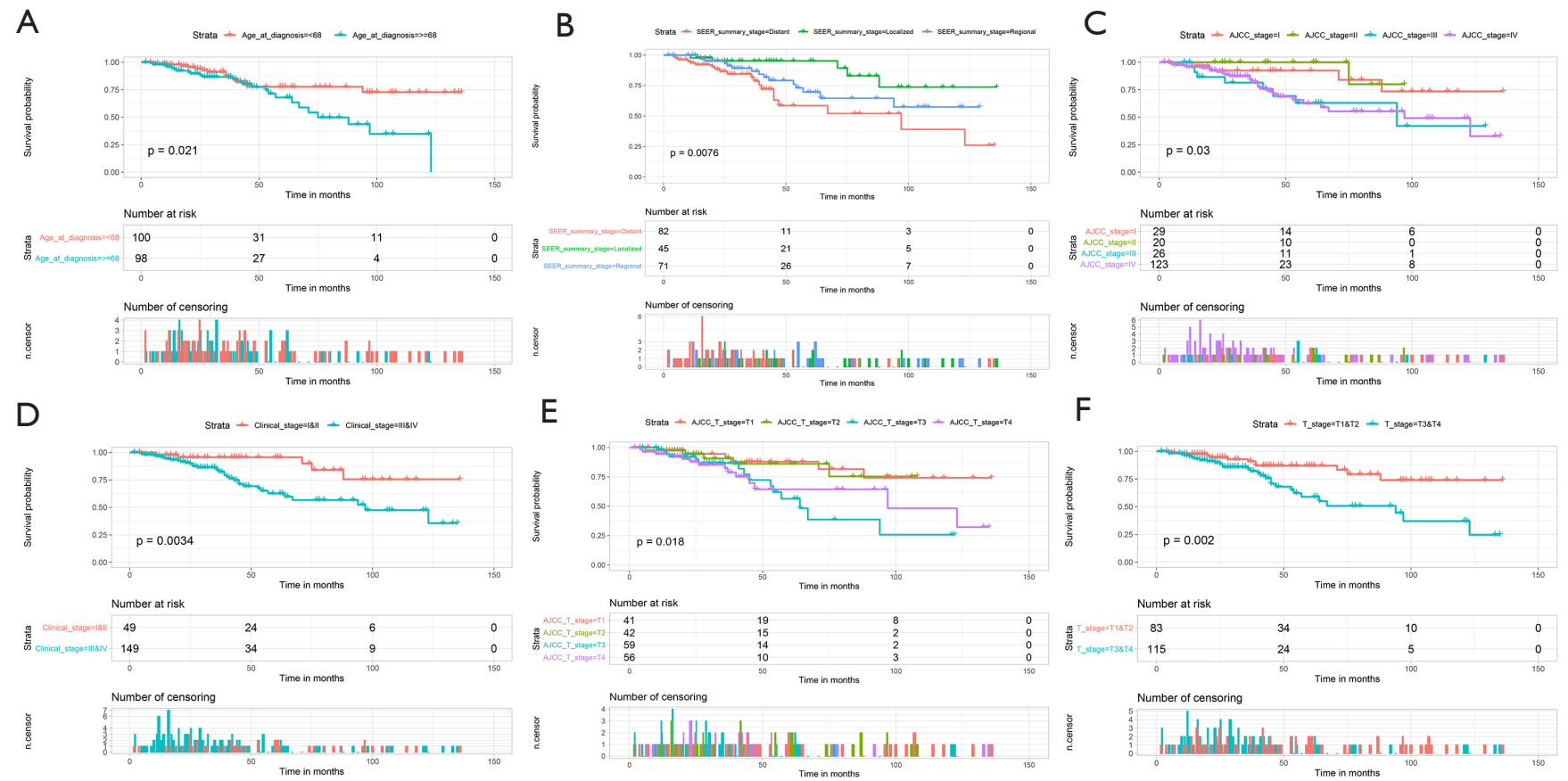

$\mathrm{F}$
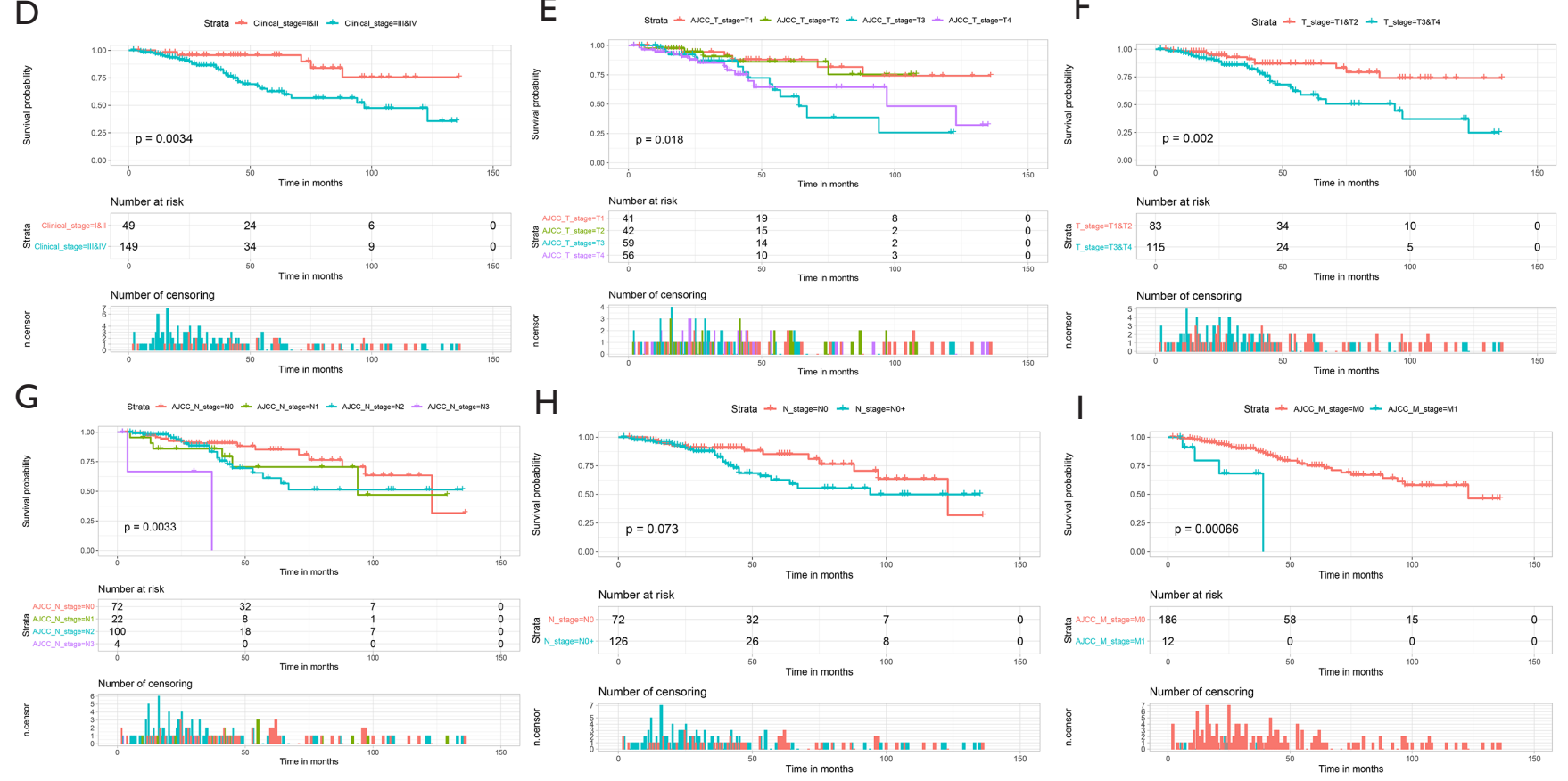

Figure 3 Kaplan-Meier curves for CSS of SDC patients stratified by (A) age at diagnosis, (B) SEER summary stage, (C,D) AJCC stage, (E,F) AJCC T stage, (G,H) AJCC N stage and (I) M stage. CSS, cancer-specific survival; SDC, salivary duct carcinoma; SEER, Surveillance, Epidemiology, and End Results; AJCC, American Joint Committee on Cancer.

of LNR in salivary gland cancer demonstrated a significant heterogeneity in prognosis by LNR (26). Therefore, LNR classification might be considered as a complementary system to TNM staging for SDC. Complementary to the previous prognostic findings on SDC, our analyses also demonstrated that the presence of multiple primary carcinomas was an independent factor for CSS for SDC patients.

There are several limitations that need to be elucidated for the present study. Though the SEER database provides a population-based cohort for the survival analysis of the rare malignancy, some inherent weaknesses still exist.
Prior studies have shown that certain pathologic features (e.g., perineural invasion, lymph-vascular invasion, extra parenchymal extension and positive surgical margins) and some genomic alterations (e.g., HER2/neu, p53 and ERbeta) are associated with OS in single institution studies (27-30). However, these factors were not available for the analysis based on the SEER database. Moreover, given that the SEER study is a retrospective collection of clinicopathological and follow-up information of cancer patients, only patients with complete information of involved variables were included in our study, which brought about selection bias. The exclusion of patients 
Table 5 Univariate and multivariate survival analysis on CSS for patients with SDC

\begin{tabular}{|c|c|c|c|c|}
\hline Variables & \multicolumn{2}{|c|}{ Univariate survival analysis } & \multicolumn{2}{|c|}{ Multivariate survival analysis } \\
\hline \multicolumn{5}{|l|}{ Age, years } \\
\hline$<68$ & Reference & & Reference & \\
\hline$\geq 68$ & $2.113(1.105-4.038)$ & $0.024^{*}$ & $1.897(1.897-3.941)$ & 0.086 \\
\hline Female & Reference & & Reference & \\
\hline Male & $1.507(0.773-3.098)$ & 0.265 & $2.022(0.888-4.604)$ & 0.094 \\
\hline \multicolumn{5}{|l|}{ Race } \\
\hline Black & Reference & 0.876 & & \\
\hline \multicolumn{5}{|l|}{ Year of diagnosis } \\
\hline 2004-2010 & Reference & & & \\
\hline 2011-2015 & $0.872(0.553-1.376)$ & 0.557 & & \\
\hline \multicolumn{5}{|l|}{ Primary site } \\
\hline Parotid gland & Reference & & & \\
\hline Submandibular gland & $1.092(0.452-2.805)$ & 0.854 & & \\
\hline \multicolumn{5}{|l|}{ Laterality } \\
\hline Left & Reference & & & \\
\hline T stage & & $0.028^{*}$ & & 0.053 \\
\hline $\mathrm{T} 1$ & Reference & & Reference & \\
\hline $\mathrm{T} 2$ & $1.171(0.356-3.855)$ & 0.795 & $1.171(0.794-2.817)$ & 0.722 \\
\hline T3 & $3.430(1.901-8.981)$ & 0.012 & $2.453(0.815-7.380)$ & 0.110 \\
\hline $\mathrm{T} 4$ & $2.794(1.065-7.332)$ & 0.037 & $3.036(1.077-8.559)$ & 0.036 \\
\hline $\mathrm{N}$ stage & & $0.017^{\star}$ & & $0.025^{\star}$ \\
\hline No & Reference & & Reference & \\
\hline $\mathrm{N} 1$ & $1.655(0.628-4.362)$ & 0.308 & $2.472(2.472-7.633)$ & 0.116 \\
\hline $\mathrm{N} 2$ & $1.750(0.857-3.575)$ & 0.125 & $1.959(0.843-4.551)$ & 0.118 \\
\hline N3 & $11.582(2.495-53.842)$ & 0.029 & $11.948(2.206-64.725)$ & 0.004 \\
\hline
\end{tabular}

Table 5 (continued) 
Table 5 (continued)

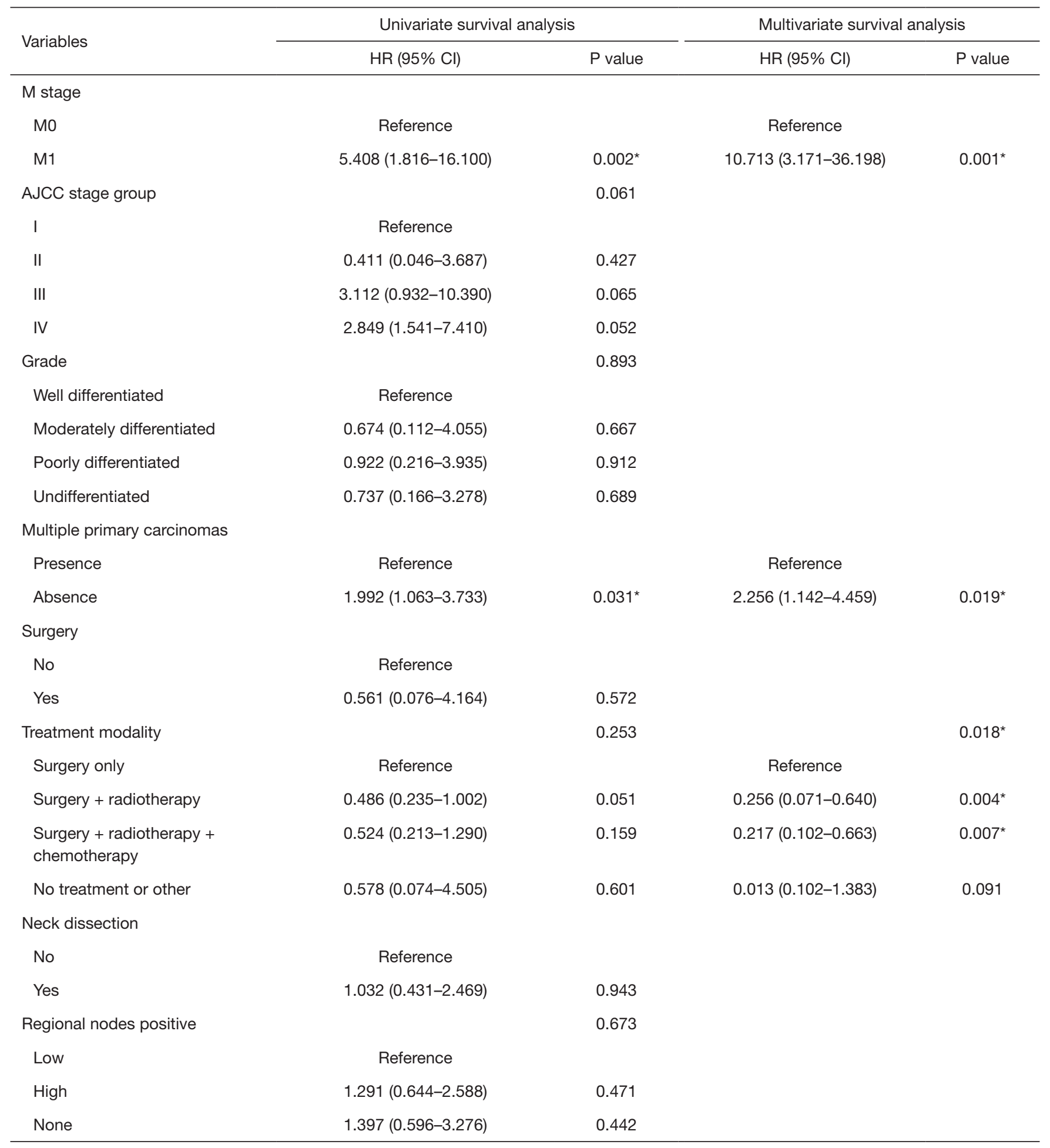

The number with * indicates statistical significance. CSS, cancer-specific survival; SDC, salivary duct carcinoma; HR, hazard ratio; CI, confidence interval; AJCC, American Joint Committee on Cancer. 
A

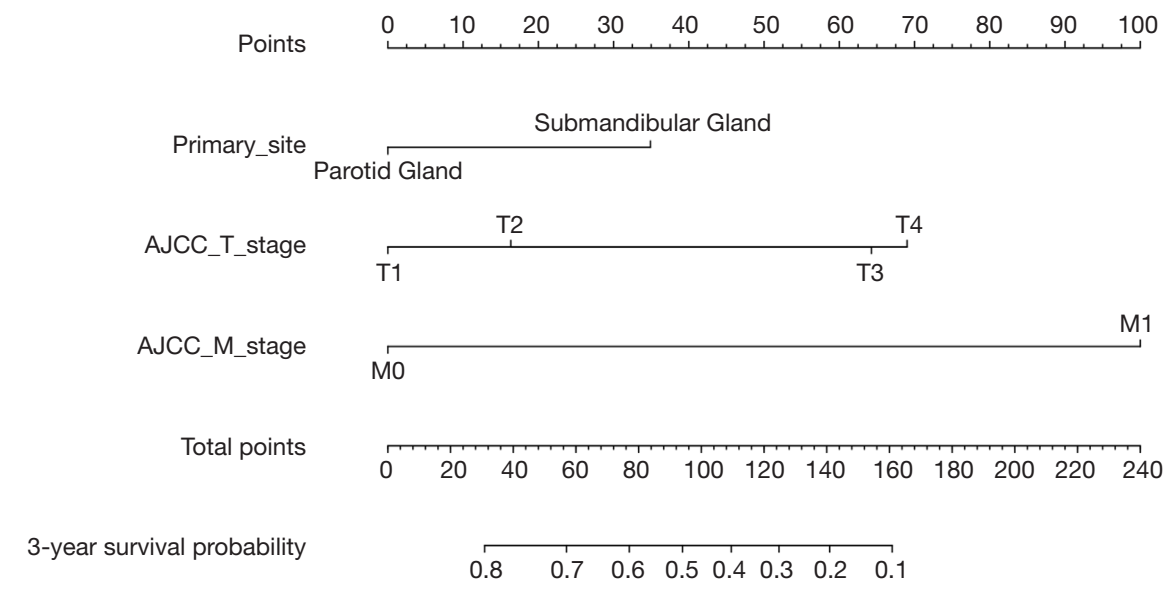

B

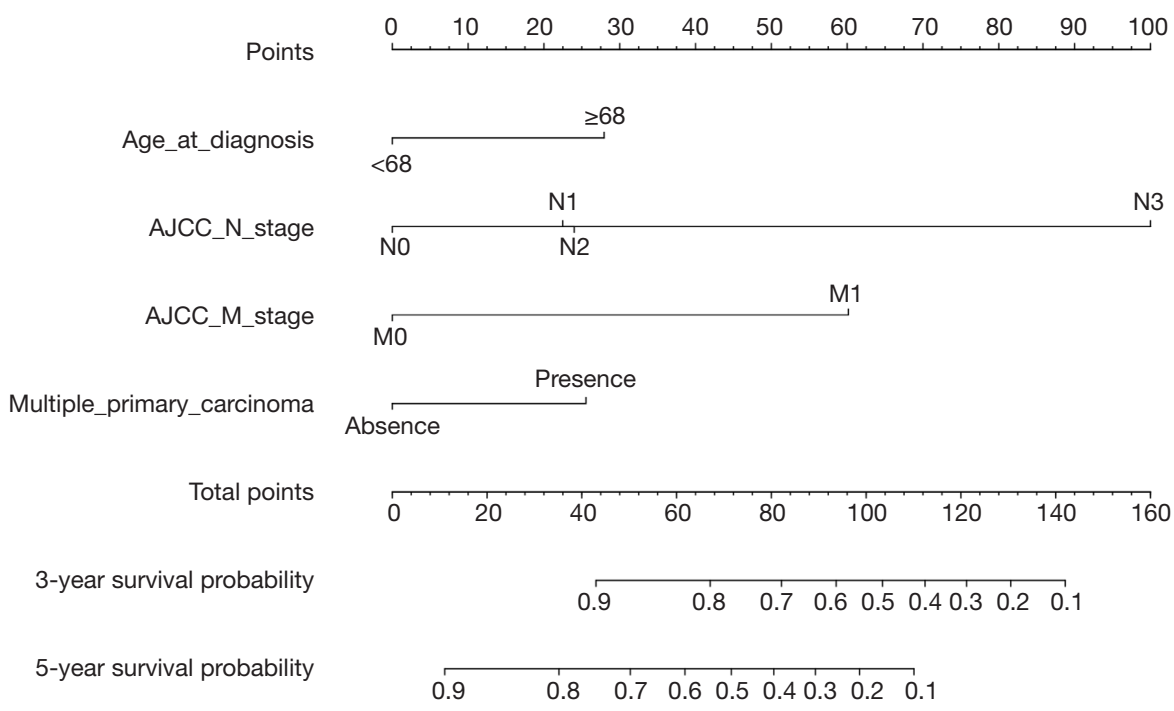

Figure 4 Nomograms for predicting 3- and 5-year OS (A) and CSS (B) of SDC patients. OS, overall survival; CSS, cancer-specific survival; SDC, salivary duct carcinoma; AJCC, American Joint Committee on Cancer.

with incomplete or missing data would also decrease the sample capacity thus reducing statistical power of our study, especially for the patients with SDC, which is a rare histological type of salivary gland cancer. Moreover, the details of adjuvant chemoradiotherapy and treatment responses could not be acquired from the SEER database, thus posing an impediment for us to evaluate the treatment regimens and facilitate the escalation of treatment modalities for SDC patients. Finally, due to the limited sample size, the reliability of our constructed nomogram could not be validated because of the lack of an independent validation cohort. Despite these limitations, our study has provided us additional information complementary to the existing criteria characterizing this rare malignancy of SDC.

\section{Conclusions}

SDC is a clinically aggressive malignancy with a high rate of tumors at the advanced stage and with regional lymph node metastases. Age at diagnosis, primary site, AJCC staging systems, LNR, the presence of multiple primary carcinomas and treatment modalities were identified as prognostic factors for SDC. A radical surgery remains the mainstay of the treatment for SDC. The use of radiotherapy or 
A
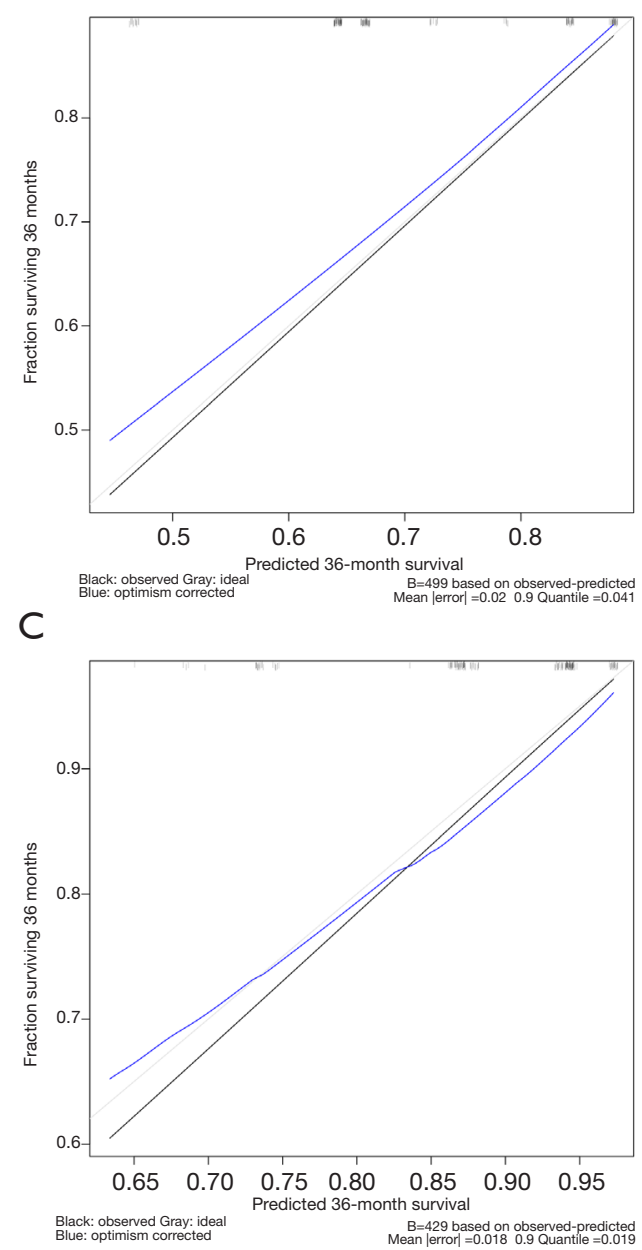

B

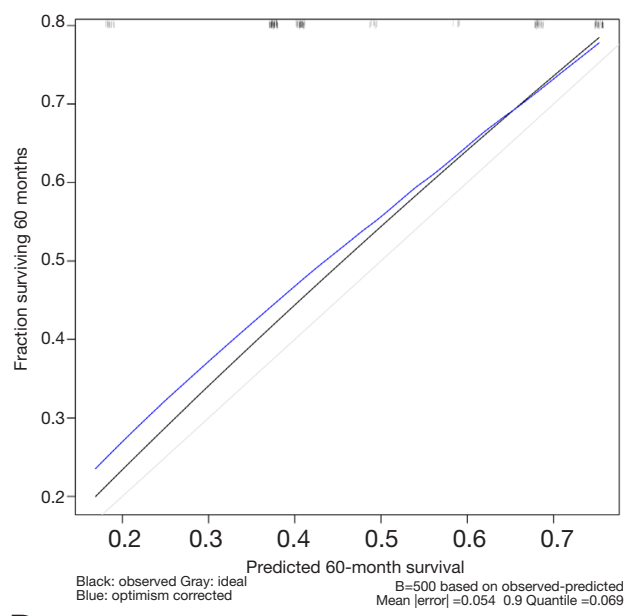

D

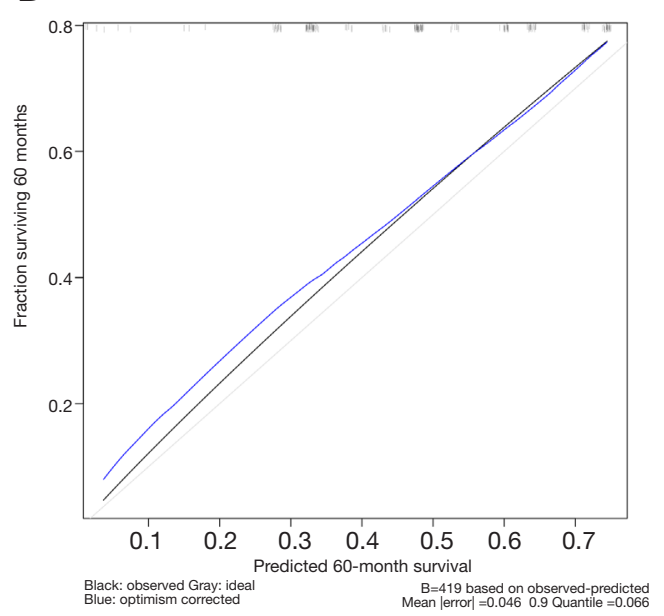

Figure 5 Calibration curves for 3-, and 5-year OS prediction (A,B) and CSS prediction (C,D) in the SDC patients. OS, overall survival; CSS, cancer-specific survival; SDC, salivary duct carcinoma.

chemotherapy was found to improve CSS remarkably. The established competing risk nomogram would facilitate the prognostic estimation of SDC patients, which needs to be further validated in more prospective studies with large sample sizes in the future.

\section{Acknowledgments}

The authors would like to thank SEER for open access to the database. In addition, we are grateful to Professor Zhou Ge from MD Anderson Cancer Center (Houston, TX, USA) for his help in reviewing the whole article to avoid any improper descriptions.

Funding: This study was supported by Jiangsu Provincial
Key Research and Development Plan (BE2017741), Jiangsu Provincial Natural Science Foundation (BK 20160051, BK20180136, BK20180138), Nanjing Cancer Clinical Medical Center, the Project of Invigorating Health Care through Science, Technology and Education Jiangsu Provincial Medical Youth Talent (QNRC2016121) and the Fundamental Research Funds for the Central Universities (14380442).

\section{Footnote}

Reporting Checklist: The authors have completed the TRIPOD reporting checklist. Available at http://dx.doi. org/10.21037/atm-20-1849 
Conflicts of Interest: All authors have completed the ICMJE uniform disclosure form (available at http://dx.doi. org/10.21037/atm-20-1849). The authors have no conflicts of interest to declare.

Ethical Statement: The authors are accountable for all aspects of the work in ensuring that questions related to the accuracy or integrity of any part of the work are appropriately investigated and resolved. The study was conducted in accordance with the Declaration of Helsinki (as revised in 2013). Since any information in the SEER database does not require explicit consent from the patients, our study was not subject to the ethical approval requirements of the institutional review board.

Open Access Statement: This is an Open Access article distributed in accordance with the Creative Commons Attribution-NonCommercial-NoDerivs 4.0 International License (CC BY-NC-ND 4.0), which permits the noncommercial replication and distribution of the article with the strict proviso that no changes or edits are made and the original work is properly cited (including links to both the formal publication through the relevant DOI and the license). See: https://creativecommons.org/licenses/by-nc-nd/4.0/.

\section{References}

1. Stodulski D, Mikaszewski B, Majewska H, et al. Parotid salivary duct carcinoma: a single institution's 20-year experience. Eur Arch Otorhinolaryngol 2019;276:2031-8.

2. Luk PP, Weston JD, Yu B, et al. Salivary duct carcinoma: Clinicopathologic features, morphologic spectrum, and somatic mutations. Head Neck 2016;38 Suppl 1:E1838-47.

3. Kim JY, Lee S, Cho KJ, et al. Treatment results of postoperative radiotherapy in patients with salivary duct carcinoma of the major salivary glands. Br J Radiol 2012;85:e947-52.

4. Salovaara E, Hakala O, Back L, et al. Management and outcome of salivary duct carcinoma in major salivary glands. Eur Arch Otorhinolaryngol 2013;270:281-5.

5. Johnston ML, Huang SH, Waldron JN, et al. Salivary duct carcinoma: treatment, outcomes, and patterns of failure. Head Neck 2016;38 Suppl 1:E820-6.

6. Breinholt H, Elhakim MT, Godballe C, et al. Salivary duct carcinoma: a Danish national study. J Oral Pathol Med 2016;45:664-71.

7. Mifsud M, Sharma S, Leon M, et al. Salivary duct carcinoma of the parotid: outcomes with a contemporary multidisciplinary treatment approach. Otolaryngol Head Neck Surg 2016;154:1041-6.

8. Boon E, Bel M, van Boxtel W, et al. A clinicopathological study and prognostic factor analysis of 177 salivary duct carcinoma patients from The Netherlands. Int J Cancer 2018;143:758-66.

9. Osborn V, Givi B, Lee A, et al. Characterization, treatment and outcomes of salivary ductal carcinoma using the National Cancer Database. Oral Oncol 2017;71:41-6.

10. Otsuka K, Imanishi Y, Tada Y, et al. Clinical outcomes and prognostic factors for salivary duct carcinoma: a multiinstitutional analysis of 141 patients. Ann Surg Oncol 2016;23:2038-45.

11. Roh JL, Lee JI, Choi SH, et al. Prognostic factors and oncologic outcomes of 56 salivary duct carcinoma patients in a single institution: high rate of systemic failure warrants targeted therapy. Oral Oncol 2014;50:e64-6.

12. Beck ACC, Lohuis $\mathrm{P}, \mathrm{Al}$-Mamgani A, et al. Salivary duct carcinoma: evaluation of treatment and outcome in a tertiary referral institute. Eur Arch Otorhinolaryngol 2018;275:1885-92.

13. Han MW, Roh JL, Choi SH, et al. Prognostic factors and outcome analysis of salivary duct carcinoma. Auris Nasus Larynx 2015;42:472-7.

14. Haderlein M, Scherl C, Semrau S, et al. Impact of postoperative radiotherapy and HER2/new overexpression in salivary duct carcinoma: a monocentric clinicopathologic analysis. Strahlenther Onkol 2017;193:961-70.

15. Gilbert MR, Sharma A, Schmitt NC, et al. A 20-year review of 75 cases of salivary duct carcinoma. JAMA Otolaryngol Head Neck Surg 2016;142:489-95.

16. Lin N, Cai JH, Xu MJ, et al. Clinicopathological analysis of 40 cases of salivary duct carcinoma. Zhonghua Kou Qiang Yi Xue Za Zhi 2019;54:416-9.

17. Jayaprakash V, Merzianu M, Warren GW, et al. Survival rates and prognostic factors for infiltrating salivary duct carcinoma: analysis of 228 cases from the Surveillance, Epidemiology, and End Results database. Head Neck 2014;36:694-701.

18. Collins GS, Reitsma JB, Altman DG, et al. Transparent reporting of a multivariable prediction model for individual prognosis or diagnosis (TRIPOD): the TRIPOD statement. BMJ 2015;350:g7594.

19. Moolgavkar SH, Chang ET, Watson HN, et al. An assessment of the cox proportional hazards regression model for epidemiologic studies. Risk Anal 2018;38:777-94.

20. Iasonos A, Schrag D, Raj GV, et al. How to build and interpret a nomogram for cancer prognosis. J Clin Oncol 
2008;26:1364-70.

21. Shinoto M, Shioyama Y, Nakamura K, et al. Postoperative radiotherapy in patients with salivary duct carcinoma: clinical outcomes and prognostic factors. J Radiat Res 2013;54:925-30

22. Huang X, Hao J, Chen S, et al. Salivary duct carcinoma: a clinopathological report of 11 cases. Oncol Lett 2015;10:337-41.

23. Al-Qahtani KH, Tunio MA, Bayoumi Y, et al. Clinicopathological features and treatment outcomes of the rare, salivary duct carcinoma of parotid gland. J Otolaryngol Head Neck Surg 2016;45:32.

24. Schmitt NC, Kang H, Sharma A. Salivary duct carcinoma: an aggressive salivary gland malignancy with opportunities for targeted therapy. Oral Oncol 2017;74:40-8.

25. Lassche G, van Boxtel W, Ligtenberg MJL, et al. Advances and challenges in precision medicine in salivary gland cancer. Cancer Treat Rev 2019;80:101906.

Cite this article as: Ran J, Zou H, Li X, Guo F, Xu W, Han W. A population-based competing risk survival analysis of patients with salivary duct carcinoma. Ann Transl Med 2020;8(21):1355. doi: 10.21037/atm-20-1849
26. Lei BW, Hu JQ, Yu PC, et al. Lymph node ratio (LNR) as a complementary staging system to TNM staging in salivary gland cancer. Eur Arch Otorhinolaryngol 2019;276:3425-34.

27. Roh JL, Cho KJ, Kwon GY, et al. Prognostic values of pathologic findings and hypoxia markers in 21 patients with salivary duct carcinoma. J Surg Oncol 2008;97:596-600.

28. Ko YH, Roh JH, Son YI, et al. Expression of mitotic checkpoint proteins BUB1B and MAD2L1 in salivary duct carcinomas. J Oral Pathol Med 2010;39:349-55.

29. Williams MD, Roberts D, Blumenschein GR Jr, et al. Differential expression of hormonal and growth factor receptors in salivary duct carcinomas: biologic significance and potential role in therapeutic stratification of patients. Am J Surg Pathol 2007;31:1645-52.

30. Jaehne M, Roeser K, Jaekel T, et al. Clinical and immunohistologic typing of salivary duct carcinoma: a report of 50 cases. Cancer 2005;103:2526-33. 